\title{
Measurement and research of environmental natural radiation dose
}

\author{
Zongxi Wan* \\ College of Earth and Environmental Sciences, Lanzhou University, Lanzhou 730000, China
}

\begin{abstract}
The living environment of human beings is being affected by various types of radiation. Among them, natural radiation has the largest dose. If the dose of natural radiation exceeds a certain threshold level, people will face health risks on multiple fronts. In this work, researchers measured the air-absorbed dose rate at 1 meter above different surface environments on campus and estimated the effective dose equivalent of environmental gamma radiation to the residents. Experiments and analyses show that the radiation level on campus was evaluated based on the measurements. The order of the amount of the radiation dose rate is granite pavement $>$ cement pavement $>$ asphalt ground. According to the experiments, the radiation content of external exposure in the campus is already slightly higher than the limit, i.e. $1 \mathrm{mSv} / \mathrm{y}$.
\end{abstract}

\section{Introduction}

The living environment of human beings has been exposed to various kinds of radiation. Generally speaking, natural radiation has the highest dose, which mainly includes cosmic rays and rays emitted by natural radioactive nuclides, such as ${ }^{40} \mathrm{~K},{ }^{232} \mathrm{Th},{ }^{238} \mathrm{U}$. This type of radiation is usually described as ambient natural radioactive radiation, also known as natural background radiation. Natural radiation can be divided into internal radiation and external radiation according to the different ways of human's exposure to radiation. Internal sources mainly include two kinds of radionuclides, radionuclides ingested by mouth and inhaled by breath. The radionuclides ingested from the mouth enter the human body through food, water and other necessities necessary to sustain human life and development, and gradually accumulate. For example, the mass fraction of uranium in the bodies of people in Western Australia are 7.5 times as that in people from other areas, as a result of eating uranium-containing lamb and kangaroo meat. Besides, there are many small particles of radioactive material suspended in the air, which are absorbed by the body through breathing, and then captured by the mucus on the larger particles' respiratory organs, which are expelled from the body in a short time. However, particles smaller than $10 \mu \mathrm{m}$, especially those smaller than $2.5 \mu \mathrm{m}$, are stuck in the alveolar walls. External sources are mainly cosmic rays and gamma radiation emitted by natural radioisotopes. Primary cosmic rays are mainly composed of positively charged high-energy particles (protons, $\alpha$ particles, etc.). When they enter the atmosphere, they react with the nuclei in the air to produce muons, neutrons, and gamma rays, which become secondary cosmic rays. The intensity of cosmic rays increases with altitude. Generally, people living in the plateau area are exposed to a higher dose of cosmic rays than those in the plain area. Surface radiation is mainly produced by the continuous decay of radionuclides in the earth's crust. The mass fraction of these nuclides in rock and soil varies greatly with the region and the type of soil, rock, and water. When human bodies are exposed to radiation, living cells are damaged. Some of them even die or mutate. However, when the radiation exceeds the threshold, human organs can be damaged or even die. Also, if the mutation of the cell repair is not perfect, its genetic information will be passed to the offspring of the cell or the cell of the offspring, and there is a risk of cancer or genetic diseases. Therefore, people must notice the danger of natural background radiation and focus on dose investigation, measurement and evaluation.

This research has received wide attention in the academic circle. A. Cardinale et al.[1] measured the exposure rate of natural gamma-ray background in representative areas of Italy by a variety of methods, and comprehensively evaluated the impact of gamma-ray radiation on the population of Italy. G. S. Gusain et al.[2] used a pocket dosimeter with an NaI detector to measure the land gamma radiation dose rate in the eastern coastal area of the state of Orissa, India, and found high concentrations of monazite and zircon minerals. S.G. Levin et al.[3] measured the natural background gamma radiation dose rate in Michigan, Colorado, and Minnesota to determine the relationship between the incidence of congenital disabilities and background radiation. M. Maharana et al.[4] determined the concentration of ${ }^{238} \mathrm{U},{ }^{232} \mathrm{Th},{ }^{40} \mathrm{~K}$ using high-resolution gamma spectrometry. After calibration and fitting, the results of the dose rate and effective dose were obtained. Tzortzis, M. et al.[5] made a comprehensive study of the natural gamma radiation levels and related dose rates of

"Corresponding author: wanzx2020@163.com 
surface soil in Cyprus using high-resolution gamma-ray spectral analysis. The results showed that the concentration of naturally occurring gamma radiation activity and associated dose rates in Cyprus were significantly lower (five times on average) than the radioactivity levels reported in other parts of the world. Sanusi et al. [6] did a terrestrial gamma radiation in the state of Selangor, Kuala Lumpur and Putrajaya, in order to obtain baseline data for environmental radiological health practices. Liu et al. [7] irradiated "Jewel" sweet potatoes with gamma radiation to investigate the relationship between dose-rate and nutrients.

In this paper, GH-102A x- $\gamma$ dose rate meter method is used to measure the absorbed dose rate of the air in the case of the campus radiation, and the overall radiation level of the campus was evaluated.

\section{Experimental methods}

\subsection{GH-102A x- $\gamma$ dose rate meter method}

In this experiment, GH-102A x- $\gamma$ dose rate meter method was used to measure the air-absorbed dose rate at 1 meter above 7 different surface environments on campus, including natural grassland, granite, asphalt, plastic venues, artificial grassland, building and hillside. Then, the effective dose equivalent of environmental gamma radiation to the residents was estimated and evaluated according to "environmental surface gamma radiation dose rate determination standard" (GB/T 14583-93)[8].

\subsubsection{Experimental instruments}

$\mathrm{GH}-102 \mathrm{~A} \mathrm{x}-\gamma$ dose rate meter is used in the experiments, as shown in Fig. 1. The measurement range $0.01 \mu \mathrm{Gy} / \mathrm{h} \sim$ $10 \mu \mathrm{Gy} / \mathrm{h}$.

The scintillator in the probe stimulated by the ray luminesces, which is accepted by the photomultiplier tube and transforms an optical signal into an electrical signal. After the A-D transformation, the voltage pulse is outputted. When the ionizing radiation field is strong, the voltage pulse frequency per unit time is high, while the pulse frequency per unit time is low when the radiation field is weak. Therefore, the radiation dose rate in the air is proportional to the measured count rate at a radiation field over a wide energy range.

The signal produced by the probe is sent to the MCU of the host through the cable for measurement and analysis. According to the actual needs of measurement, certain parameters are selected and set (such as sampling time, sampling times, and cycle times, etc.). After that, start the measurement, and the final result would be automatically shown. (mean value, standard deviation, measurement time, and date)

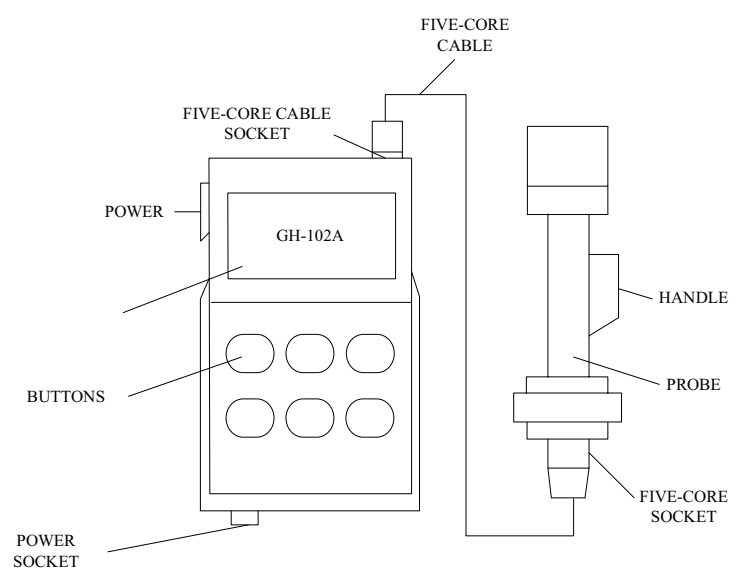

Fig 1. Schematic diagram of the instrument

\subsubsection{Experimental principle}

The principle of $\mathrm{GH}-102 \mathrm{~A} \mathrm{x}-\gamma$ dose rate meter: The incoming radiation loses and deposition energy in the scintillator, causing the ionization excitation of the atoms (or ions, molecules) in the scintillator, and then the stimulated particles deexcite and emits the scintillation photon whose wavelength is close to that of visible light. The scintillation photon is transmitted into the photoelectrode of the photomultiplier tube and emits photoelectrons. The photoelectrons are accelerated because of the interactions of strong electric fields in dynodes and strike the next dynode, producing more photoelectrons and multiplying them until they finally reach the anode and produce signals in the output circuit.[9] The process is shown in Fig. 2. When the ionizing radiation field is strong, the voltage pulse frequency per unit time is high, and when the radiation field is weak, the pulse frequency per unit time is low. Therefore, the radiation dose rate in the air is proportional to the measured count rate at a radiation field over a wide energy range.

\section{Experimental steps}

\subsection{Measurement sites}

In this experiment, several sites were selected on the campus of Tsinghua University for the measurement of radiation air-absorbed dose rate. 7 sites were selected in total, namely natural grassland, granite ground, asphalt pavement, plastic field, artificial grassland, building and hillside.

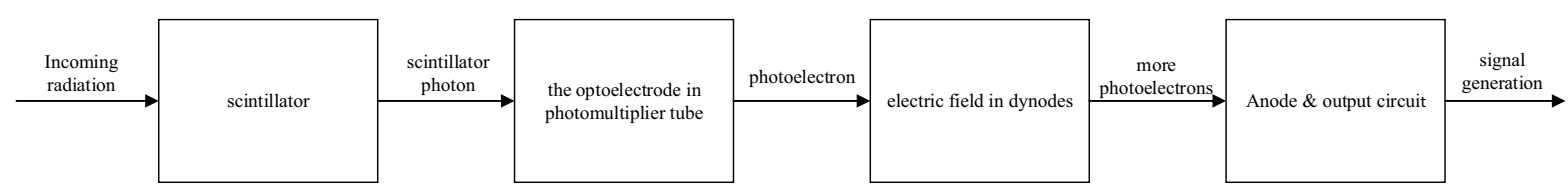

Fig 2. The flow chart of using GH-102A $x-\gamma$ dose rate meter 


\subsection{Instrument settings}

\subsubsection{Sampling time}

Sampling time is the minimum time interval for the measurement in seconds. The selection range is $1 \mathrm{~s}-120 \mathrm{~s}$. In this experiment, the sampling time is set to $30 \mathrm{~s}$.

\subsubsection{Sampling number}

The sampling number is the sampling number of a single cycle. The selection range is 1-100 times. In this experiment, the sampling number is set to 10 times.

\subsubsection{Cycle index}

The cycle index is the number of repetitions in a single cycle. The selection range is 1-150 times. In this experiment, the cycle index is set to 1 time.

\subsection{Experimental measurement}

The probe was fixed on a tripod to make sure that the probe is 1 meter high above the ground. Then the signal line was connected. After that, the dose rate meter was turned on and preheated for $15 \mathrm{~min}$. The measurement formally started after the parameters were set properly.

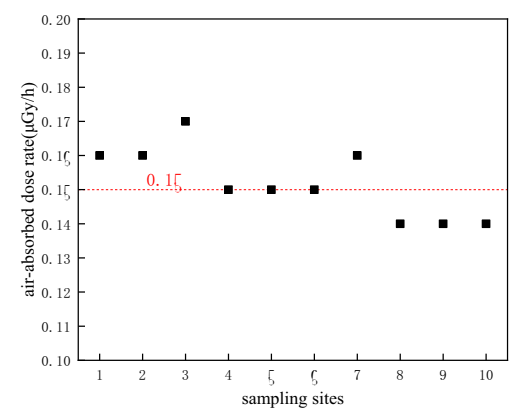

Fig 3. Measurement results of the NO.4 teaching building

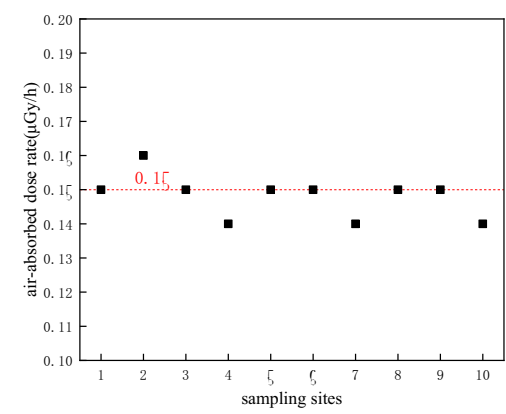

Fig 4. Measurement results of the granite pavement
The method of moving measurement was adopted, and the position was changed after the data of a site had been read. Different grid methods were adopted according to the different measurement sites, such as chessboard means and serpentine means, to evenly distribute the sampling points in the site. The instrument readings and average values were recorded on the spot. (Natural background radiation was not considered in this experiment.)

\section{Experimental results, calculations and analysis}

\subsection{Experimental results}

In this experiment, 7 measuring sites were selected on the campus of Tsinghua University, including in the No.4 teaching building (indoor), the granite pavement in front of the liberal arts library, the lover's slope (a slope covered with natural grass), the grass under the dormitory building, the asphalt pavement, the running track of the west playground and the football field of the west playground. At each site, 10 sampling points were set to measure the air-absorbed dose rate. The measurement results are shown in Fig. 3-9.

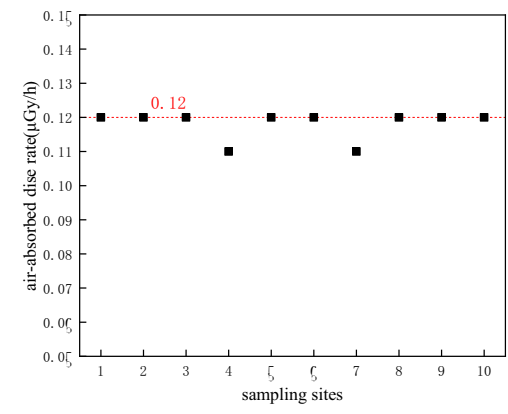

Fig 5. Measurement results of the lovers' slope

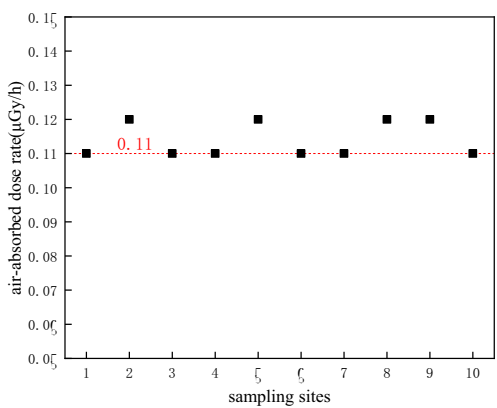

Fig 6. Measurement results of the grassland 


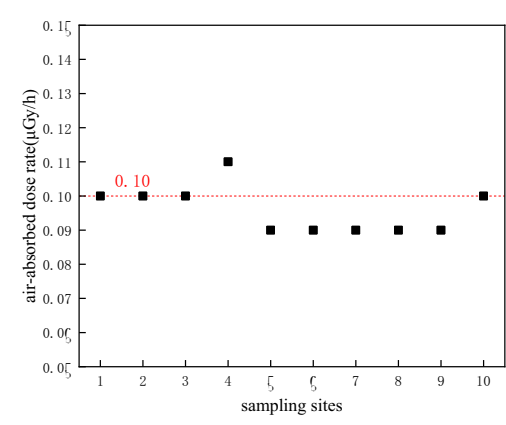

Fig 7. Measurement results of the asphalt pavement

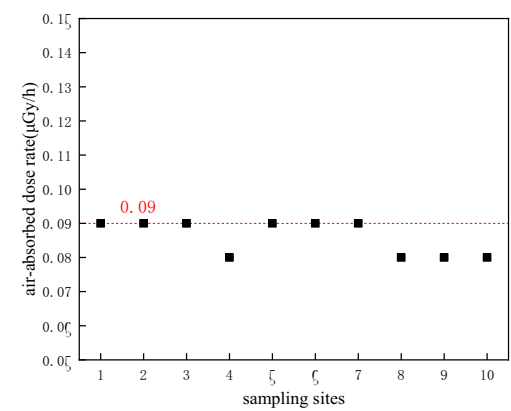

Fig 8. Measurement results of the plastic track

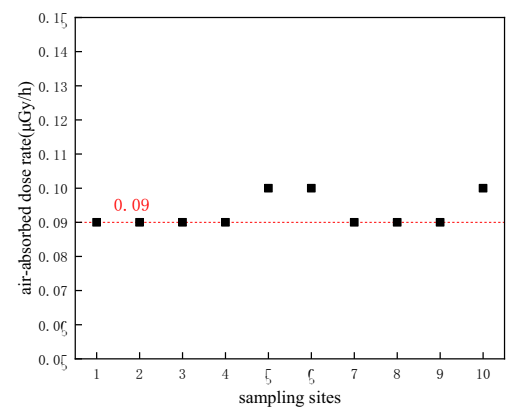

Fig 9. Measurement results of the artificial turf

As shown in Fig. 3-9, the air-absorbed gamma dose rates of the NO.4 teaching building and granite pavement are about $0.15 \mu \mathrm{Gy} / \mathrm{h}$; the air-absorbed gamma dose rate of the lovers' slope is about $0.12 \mu \mathrm{Gy} / \mathrm{h}$; the air-absorbed gamma dose rate of the grassland under the dormitory building is about $0.11 \mu \mathrm{Gy} / \mathrm{h}$; the air-absorbed gamma dose rate of asphalt pavement is about $0.10 \mu \mathrm{Gy} / \mathrm{h}$; the air-absorbed gamma dose rates of the plastic track and the artificial turf are about $0.09 \mu \mathrm{Gy} / \mathrm{h}$. The fluctuation ranges of the measurement data at 10 sampling sites of each of the 7 locations in the university were rather small, indicating that the measurement results were accurate and the error was small.

\subsection{Calculation of effective dose equivalent of radiation}

According to the standard for the measurement of environmental surface radiation dose rate $(\mathrm{GB} / \mathrm{T}$ 14583-93)[8], the effective dose equivalent to residents produced by environmental radiation can be estimated by the following formula:

$$
H_{e}=D_{r} \times K \times t
$$

In the formula,

$H_{e}$ : effective dose equivalent, $\mathrm{Sv}$

$D_{r}$ : air-absorbed dose rate of environmental surface gamma radiation

$K$ : the ratio of effective dose equivalent rate to air-absorbed dose rate (This standard adopts $0.7 \mathrm{~Sv} / \mathrm{Gy}$ )

$t$ : residence time in the environment, $\mathrm{h}$

The time of people staying indoors and outdoors are assumed to be $4 \mathrm{~h}$ and $24 \mathrm{~h}$ each day. In this experiment, people are assumed to stay for $40 \mathrm{~min}$ in each of the other sites, so the radiation amount people received per day is: (using the maximum result of the measurement). The quantity of radiation people received in a year is $\mathrm{He}$ is $1.059 \mathrm{mSv}$.

\subsection{Data analysis}

According to ICRP recommendation no. 60 in 1990, the annual acceptable radiation dose limit set by the international commission on radiation protection (ICRP) for the public is $1 \mathrm{mSv}$ [10]. And the radiation content of external exposure at Tsinghua University is already slightly higher than the limit. This experiment did not measure the radiation level of internal exposure. For example, the measurement of radon concentration was not carried out. Therefore, the actual total natural background radiation level should be higher than $1 \mathrm{mSv}$. Clarke, chairman of the ICRP radiation, said that the protection system should be the key to personal protection. As for a controlled source of radiation, if individuals exposed to the largest dose of radiation acquire fully protection, the health risk of the individuals is slight, so that the total risk is slight, no matter how many people are exposed to the radiation. This principle not only works for the public but also works for the officers. The personal average annual effective dose of radiation protection degree can be shown in Table 1 [11]. As the figure shows, the degree of effective concern of individuals at Tsinghua University has reached level 3. It is necessary to measure the total radiation level, including the level of internal radiation. After that, people should take measures according to the radiation level to reduce the radiation level on campus.

Table 1 Classification of annual effective concern level of individuals

\begin{tabular}{ccc}
\hline Level of concern & explanation & Dose level/(mSv·year-1) \\
\hline 1 & serious & $>100($ general natural radiation level $)$
\end{tabular}




\begin{tabular}{ccc}
$\mathbf{2}$ & high & $>10($ general natural radiation level $)$ \\
$\mathbf{3}$ & common & $1 \sim 10($ typical natural radiation level $)$ \\
$\mathbf{4}$ & low & $>0.1($ general natural radiation level $)$ \\
$\mathbf{5}$ & tiny & $>0.01($ general natural radiation level $)$ \\
$\mathbf{6}$ & negligible & $<0.01($ general natural radiation level $)$ \\
\hline
\end{tabular}

According to the experimental results, it can be inferred that the radiation levels of different building materials and different environments are different because the different contents of radioactive substances such as isotopes in different materials lead to differences in the results. In general, the gamma radiation air-absorbed dose rate in the newly decorated NO.4 teaching building is rather high because of the relatively high content of radioactive elements in the material. The exposed basic rocks also lead to higher measurement results. As the data shows, the order of the amount of the radiation dose rate is granite pavement $>$ cement pavement $>$ asphalt ground.

By calculating the standard deviation of ten measurements, it can be inferred that the overall data of each measurement does not fluctuate much. It provides the corresponding guarantee for the numerical validity of this measurement experiment.

\section{Conclusions}

In this paper, $\mathrm{GH}-102 \mathrm{~A} \mathrm{x}-\gamma$ dose rate meter method is used to measure the absorbed dose rate of the air in the case of the campus radiation, and the overall radiation level of the campus was evaluated. By calculating the standard deviation of ten measurements, it can be inferred that the overall data of each measurement does not fluctuate much. It provides the corresponding guarantee for the numerical validity of this measurement experiment.

Experiments show that the gamma radiation air-absorbed dose rate in the newly decorated NO.4 teaching building is rather high because of the relatively high content of radioactive elements in the material. The exposed basic rocks also lead to higher measurement results. The order of the amount of the radiation dose rate is granite pavement $>$ cement pavement $>$ asphalt ground.

According to the experiments, the radiation content of external exposure at Tsinghua University is already slightly higher than the limit, $1 \mathrm{mSv} / \mathrm{y}$.

The author thanks Dr. Ma Yugao for his patient guidance.

\section{References}

1. A. Cardinale, L. Frittelli, G. Lembo, F. Gera, O. Ilari, Studies on the Natural Background Radiation in Italy, Health Physics, 20 285-296.

2. G.S. Gusain, B.S. Rautela, S.K. Sahoo, T. Ishikawa, G. Prasad, Y. Omori, A. Sorimachi, S. Tokonami, R.C. Ramola, Distribution of terrestrial gamma radiation dose rate in the eastern coastal area of Odisha, India, Radiation Protection Dosimetry, 152 42-45.

3. S.G. Levin, R.K. Stoms, Natural background gamma radiation dose rate measurements in Michigan, Colorado, and Minnesota, Am J Public Health Nations Health, 59 102-109.

4. M. Maharana, N. Krishnan, D. Sengupta, Spatial distribution of gamma radiation levels in surface soils from Jaduguda uranium mineralization zone, Jharkhand, India, using $\hat{\mathrm{I}}^{3}$-ray spectrometry, and determination of outdoor dose to the population, 35 (2010) 235.

5. Tzortzis, M., A comprehensive study of natural gamma radioactivity levels and associated dose rates from surface soils in cyprus, Radiation Protection Dosimetry, 109 217-224.

6. M.S.M. Sanusi, A.T. Ramli, H.T. Gabdo, N.N. Garba, A. Heryanshah, H. Wagiran, M.N. Said, Isodose mapping of terrestrial gamma radiation dose rate of Selangor state, Kuala Lumpur and Putrajaya, Malaysia, Journal of Environmental Radioactivity, 135 67-74.

7. J. Liu, P. Miller, P.A. Loretan, Gamma radiation dose rate and sweet potato quality, Journal of Food Quality, 12 (2007) 369-376.

8. Specification for the determination of environmental surface ground dose rates (in Chinese), in, 1993.

9. Y. Zhang, G. Zeng, research trends of scintillators and scintillation detectors, science and technology information (in Chinese), (2007) 81+83.

10. Y. Zhou, dosimetry and recommended dose limits in the 1990 recommendations of the international commission on radiological protection, radiation protection communications (in Chinese), (1992) $1-10$.

11. Reger $\mathrm{H}$ Clarke. Progress towards new recommendations from the international commission on radiological protection, Radiation Protection, (2000) 321-326. 